\title{
Langfristig gute Wirkung und Verträglichkeit
}

Über die Jahre sind die Ziele der MS-Therapie immer anspruchsvoller geworden. In die Bewertung des Nutzens einer Behandlung müssen Patienten intensiv mit einbezogen werden.

Die moderne und nachgewiesenermaßen effektive Behandlung der schubförmigen multiplen Sklerose (MS) beginnt mit der Einführung von Interferon beta-1b (IFN $\beta$-1b) in den frühen 90er Jahren, berichtete Univ.Prof. Dr. Siegrid Fuchs, Graz. Diese Substanz hat wie andere $\beta$-Interferone sowie Glatirameracetat (GA) die Schubraten über 2 Jahre in den Zulassungsstudien um gute $30 \%$ verringert und bei 30 bis $40 \%$ der Patienten Schubfreiheit erreicht. Allerdings, so Fuchs, haben wir nicht nur Schübe gezählt, sondern auch das Fortschreiten der Behinderung erfasst und damit das, worum es eigentlich geht. Mit IFN $\beta-1 a$ (zum Beispiel Rebif ${ }^{\oplus} 44 \mu \mathrm{g}$ ) konnte die EDSS-Progression (Expanded Disability Status Scale) signifikant reduziert werden. Bei IFN $\beta$-1b und GA war der Effekt nicht signifikant.

Über die Jahre sind die Ziele der MS-Therapie immer anspruchsvoller geworden. Man will heute Freiheit von klinischer und MR-Aktivität, zusammengefasst als Freiheit von Krankheitsaktivität erreichen. Das neueste Schlagwort lautet NEDA (No Evidence of Disease Activity). Allerdings ist die umfassende Bewertung der Erkrankung mit NEDA hauptsächlich in Studien praktikabel und eignet sich eher weniger für die tägliche Praxis.

In die Bewertung des Nutzens einer Behandlung müssen Patienten intensiv mit einbezogen werden. Denn ihre Vorstellungen unterscheiden sich oft von denen des Arztes. Auch die Bereitschaft, therapiebedingte Risiken einzugehen, variiert abhängig von der jeweiligen Persönlichkeit des Betroffenen. Bei den meist jungen Patienten spielen die Familiensituation und Themen wie Kinderwunsch oder Schwangerschaft eine wichtige Rolle. Die neuen
MS-Therapeutika können sich in dieser Hinsicht durchaus als problematisch erweisen, da sie mit erhöhter Infektanfälligkeit, Gefahr von Virusinfektionen oder der progressive multifokale Leukenzephalopathie (PML) einhergehen können, erklärte Fuchs. Vor allem aber fehlen bei diesen Substanzen noch die Erfahrungen über eine langfristige Anwendung.

\section{Herausforderung transkulturelle Arzt-Patienten-Beziehung}

Mit der Migration erwachsen der medizinischen Versorgung neue Herausforderungen. An der Medizinischen Universität in Innsbruck hat man entsprechend bereits vor drei Jahren eine transkulturelle MS-Ambulanz eingerichtet, erklärte Univ.-Prof. Dr. Thomas Berger. Von 2800 MS-Patientinnen in Tirol stammen etwa 3,6\% aus der Türkei, etwa der gleiche Anteil kommt aus Südosteuropa. Im Zusammenhang von Migration und Gesundheit spielen Aspekte wie der kulturelle Hintergrund der Migranten mit Sprache, Religion, Traditionen sowie soziale Bindungen eine bedeutende Rolle. Wichtig ist auch der Migrationsprozess als kritisches Lebensereignis sowie die Situation, mit der Menschen nach der Flucht konfrontiert werden wie Diskriminierung, Status als Minorität, Konfrontation mit einer fremden Kultur. Nicht zu vernachlässigen sind die soziale Lage der Betroffenen bezüglich Ressourcen, Bildung, Beruf, Geschlecht.

Als konkrete Herausforderungen in der Ambulanz nannte Berger sprachliche Barrieren oder mangelnde Kenntnisse über Gesundheits- und Versicherungssystem in Österreich. Oftmals haben Migranten eine andere Vorstellung, was Gesundheit, Krankheit und den Umgang damit bedeutet. Für manche bedeutet Krankheit eine gottgegebene Strafe oder gilt als schicksalshaft.

Die Sprachbarriere kann Probleme und Frust auf beiden Seiten hervorrufen. Manche Patienten erscheinen mit einem Kind oder dem Ehepartner als Laiendolmetscher. Dass dabei die Mitteilung speziell intimer Nachrichten schwierig ist, ist nachvollziehbar. Oft weiß man nicht, was letztlich an Information übermittelt wurde. Gerade Kinder von MS-Patienten mit Migrationshintergrund, die einerseits dolmetschen und sich gleichzeitig am Behandlungsprozess ihrer Mutter oder Vater beteiligen müssen, stehen manchmal unter enormem Druck. Professionelle externe Dolmetscher weisen zumindest nicht die Empathie gegenüber dem Kranken aus wie Familienmitglieder.

Sehr hilfreich ist eine bilinguale medizinische Betreuung, entweder persönlich durch einen "kultureigenen“ Arzt oder Ärztin, andererseits mit Hilfe von Informationsmaterialien in der jeweiligen Landessprache. Damit bietet sich die Chance, das transkulturelle Gefälle zum Wohle von Arzt und Patienten zumindest teilweise zu überwinden. Auch wenn die Betreuung von MS-Patienten mit Migrationshintergrund eine echte Herausforderung bedeutet, kann sie für jemanden, der sich für Lebensgeschichten anderer Menschen interessiert, auch zu einem großen Gewinn werden, führte Berger abschließend aus.

psychopraxis. neuropraxis

2016 $\cdot 19: 97$

DOI 10.1007/s00739-016-0320-3

Online publiziert: 21. April 2016

(c) Springer-Verlag Wien 2016

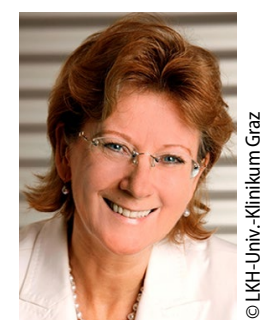

Univ.-Prof. Dr. Siegrid Fuchs

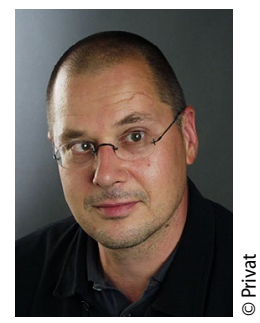

Univ.-Prof. Dr. Thomas Berger
Quelle:

Lunchsymposium „Reise in das Universum der Therapie der Multiplen Sklerose" veranstaltet von Merck anlässlich der 13. Jahrestagung der Österreichischen Gesellschaft für Neurologie am 16. März 2016 in Innsbruck/Martin Bischoff 